\title{
In situ synthesis of gold nanostars within liposomes for controlled drug release and photoacoustic imaging
}

\author{
Malathi Mathiyazhakan ${ }^{1}$, Paul Kumar Upputuri ${ }^{1}$, Kathyayini Sivasubramanian ${ }^{1}$, Ashish Dhayani ${ }^{2}$, \\ Praveen Kumar Vemula ${ }^{2,3}$, Peichao Zou ${ }^{4}$, Kanyi $\mathrm{Pu}^{1}$, Cheng Yang ${ }^{4}$, Manojit Pramanik ${ }^{1}$ and \\ Chenjie $\mathrm{Xu}^{1,5^{*}}$
}

\begin{abstract}
This report describes the design and synthesis of gold nanostars (AuNSs) containing liposomes by the in situ reduction of gold precursor, $\mathrm{HAuCl}_{4}$ (pre-encapsulated within the liposomes) through HEPES diffusion and reduction. Compared with the conventional process that encapsulates the pre-synthesized gold nanoparticles into liposomes during the thin-film hydration step, this facile and convenient method allows the formation and simultaneous encapsulation of AuNSs within liposomes. The absorption spectra of AuNSs can be tuned between visible and near infra-red (NIR) regions by controlling the size and morphology of AuNSs through varying the concentrations of $\mathrm{HAuCl}_{4}$ and $\mathrm{HEPES}$. As a proof of concept, we demonstrate the synthesis of AuNSs with a maximum absorbance at $803 \mathrm{~nm}$ within the temperature-sensitive liposomes. These liposomes can produce stronger photoacoustic signals (1.5 fold) in the NIR region than blood. Furthermore, when there are drugs (i.e., doxorubicin) within these liposomes, the irradiation with the NIR pulse laser will disrupt the liposomes and trigger the $100 \%$ release of these pre-encapsulated drugs within 10 seconds. In comparison, there is neglectable contrast enhancement or minor release $(10 \%)$ of drugs for the pure liposomes under the same conditions. Finally, cell experiment shows the potential therapeutic application of this system.
\end{abstract}

Keywords: gold nanostars, light sensitive liposomes, controlled drug release, photoacoustic imaging

\section{INTRODUCTION}

Light sensitive liposomes have attracted increasing attention due to their ability to induce controlled drug release in terms of time, location, and dosage $[1,2]$. The most convenient and straightforward way to synthesize such liposomes is to incorporate photosensitizing components within the liposomes. They can be lipid-like chemicals that undergo conformational change under laser irradiation or nanoparticles that absorb light and convert the photon energy into thermal energy [3,4]. Gold nanoparticles are one of the most attractive photosensitizing agents due to their biocompatibility and distinctive optical properties. For example, liposomes containing gold nanoparticles can be triggered with a $532 \mathrm{~nm}$ pulse laser to release the encapsulated fluorescent dyes and gold nanorods with a characteristic surface plasmon band at $792 \mathrm{~nm}$ (longitudinal) can be incorporated into lipid layers to provide a theranostic platform for siRNA delivery to the tumour [5-7]. Currently, the integration of gold nanoparticles and liposomes is mainly achieved through hydrating the lipid film with the aqueous solution containing pre-synthesized nanoparticles. This procedure is simple and convenient, but it usually suffers from the low encapsulation of nanoparticles [8].

This article reports a protocol for the in-situ synthesis of gold nanoparticles within liposome vehicles as an alternative strategy (Scheme 1). Specifically, it starts with the formulation of chloroauric acid $\left(\mathrm{HAuCl}_{4}\right)$ containing liposomes before reducing the encapsulated $\mathrm{HAuCl}_{4}$ to gold nanoparticles using a mild reducing agent (i.e., 2-[4-(2-hydroxyethyl)-1-piperazinyl]ethanesulfonic acid (HEPES)) $[9,10]$. As a proof-of-concept, gold nanostars (AuNSs)

\footnotetext{
${ }^{1}$ School of Chemical \& Biomedical Engineering, Nanyang Technological University, 62 Nanyang Drive, Singapore

${ }^{2}$ Institute for Stem Cell Biology and Regenerative Medicine (inStem), GKVK-Campus, Bellary Road, Bangalore, India

${ }^{3}$ Ramalingaswami Re-Entry Fellow, Department of Biotechnology, New Delhi 110003, India

${ }^{4}$ Division of Energy \& Environment, Graduate School at Shenzhen, Tsinghua University, Shenzhen 518055, China

${ }^{5}$ NTU-Northwestern Institute for Nanomedicine, Nanyang Technological University, 50 Nanyang Avenue, Singapore

*Corresponding author (email: cjxu@ntu.edu.sg)
} 


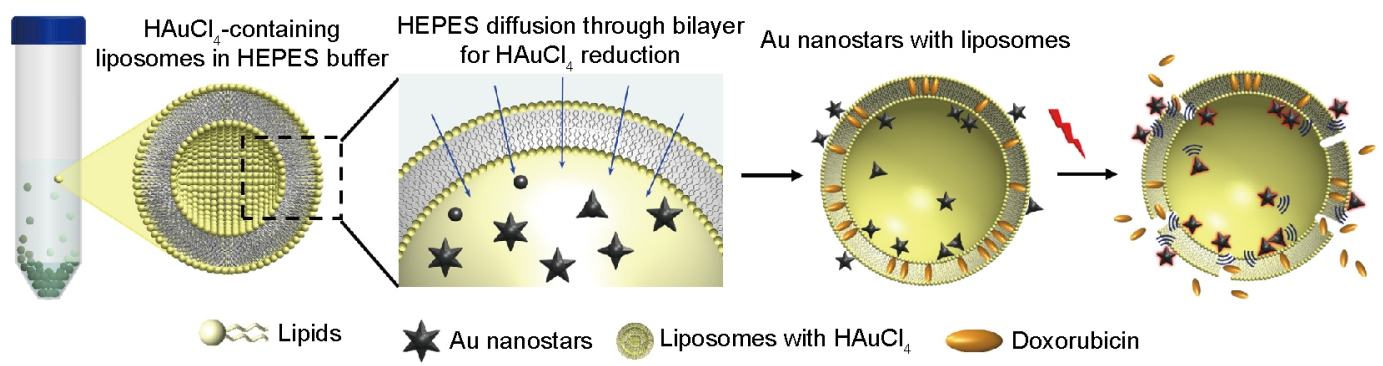

Scheme 1 Schematic of the synthesis of AuNS-liposome complex by diffusion controlled method and its use in light-controlled drug release.

with the maximum absorbance in the near-infrared (NIR) wavelength range (i.e., $803 \mathrm{~nm}$ ) were synthesized within the temperature-sensitive liposomes. These AuNSs containing liposomes showed stronger photoacoustic signals ( 1.5 fold $)$ in the NIR region than fresh blood. Furthermore, when there were drugs (i.e., doxorubicin (Dox)) within these liposomes, the irradiation with the NIR pulse laser would disrupt the liposomes and trigger the $100 \%$ release of preencapsulated Dox within 10 seconds. In contrast, there was negletable contrast enhancement and minor laser-triggered release (10\%) for the liposomes without AuNSs. Finally, cancer cells were treated with these Dox-containing AuNS-liposomes with or without the laser stimulation. The cell viability assay showed that over $90 \%$ cells died only under the NIR laser treatment. Taking together, this article reported a convenient and efficient way to prepare the AuNS-liposome complex for both photoacoustic imaging and controlled drug delivery.

\section{MATERIALS AND METHODS}

1,2-Dipalmitoyl-sn-glycero-3-phosphocholine (DPPC), 1-palmitoyl-2-hydroxy-sn-glycero-3 phospho-choline (MPPC) and 1, 2-distearoyl-sn-glycero-3-phosphoethanol-amine- $N$-[carboxy (polyethylene glycol)-2000] (ammonium salt) (DSPE-PEG2000) were purchased from Avanti Polar Lipids (Alabama, US). HAuCl4 was purchased from ACROS Organics. Fresh mouse blood was collected from animal facility center, Nanyang Technological University. All other chemicals unless otherwise mentioned were purchased from Sigma-Aldrich. Deionized (DI) water was purified by a Millipore Milli-DI water purification system.

\section{Synthesis of liposomes encapsulating $\mathrm{HAuCl}_{4}$ or $\mathrm{HAuCl}_{4} / \mathrm{Dox}$} Liposomes encapsulating $\mathrm{HAuCl}_{4}$ were synthesized by thin film hydration method [11,12]. Briefly, the constituent phospholipids (DPPC, MPPC and DSPE-PEG2000) were mixed and dissolved in chloroform in a molar ratio of 86:10:4 (15.9 mg: $1.3 \mathrm{mg}: 2.8 \mathrm{mg}$ ). The mixture was evaporated in a rotary evaporator to form a thin lipid layer, which was then re-hydrated by solution containing $\mathrm{HAuCl}_{4}$ $\left(20 \mathrm{mmol} \mathrm{L}^{-1}\right)$ for AuNS-liposome complex. Then the synthesized liposomes were extruded using a hand-held mini-extruder. Liposomes were purified from unencapsulated $\mathrm{HAuCl}_{4}$ by centrifuging the liposomes at 10,000 $\times \mathrm{g}$ at $4^{\circ} \mathrm{C}$ for $60 \mathrm{~min}$. To prepare liposomes containing $\mathrm{HAuCl}_{4} / \mathrm{Dox}, 2 \mathrm{mg}$ of Dox and $1 \mu \mathrm{L}$ of triethylamine (TEA) were added to the chloroform along with the lipids as above [13]. The rest procedure was the same as the above.

\section{Diffusion controlled reduction of $\mathrm{HAuCl}_{4}$ in liposomes with HEPES}

After the removal of unencapsulated $\mathrm{HAuCl}_{4}$, the liposomes were reconstituted with $5 \mathrm{~mL}$ solution containing 1 mol L ${ }^{-1}$ HEPES. The mixture was left in dark for one hour to allow the reduction of $\mathrm{HAuCl}_{4}$ by HEPES [14]. Then the liposomes were purified by gel filtration chromatography using PD-10 desalting column (GE Healthcare) to remove the excess HEPES. Purified samples were then stored at $4^{\circ} \mathrm{C}$ until further use.

\section{Characterization}

Hydrodynamic diameter and zeta potential of the prepared liposomes were measured with dynamic light scattering (DLS, ZetaPALS analyser, BIC). Each sample was measured at least 6 times. The UV-vis absorbance was measured with UV-visible spectrophotometer (UV-2450, Shimadzu).

For transmission electron microscopy (TEM), $10 \mu \mathrm{L}$ of sample solution was placed on 200 mesh formvar carbon coated copper grids (FCF-200-Cu, Electron Microscopy Sciences) and air-dried. The sample was then negatively stained using 3\% uranyl acetate for $1.5 \mathrm{~min}$ followed by washing with DI water. Finally, the grid samples was air-dried overnight before imaging with FEI Tecnai G2 Spirit Bio-TWIN electron microscope, operated at $120 \mathrm{kV}$.

For high resolution TEM (HRTEM) imaging, the liposomes were lysed with Triton X-100 and AuNSs were collected after centrifuging the samples at $10,000 \times \mathrm{g}$ for 15 
$\min$ at $4^{\circ} \mathrm{C}$. AuNSs were then re-suspended in DI water and $5 \mu \mathrm{L}$ of this suspension was placed on 200 mesh formvar carbon coated copper grids (FCF-200-Cu, Electron Microscopy Sciences). The grids were completely dried in a dry cabinet and were imaged using TEM (FEI G2 spirit, $120 \mathrm{kV}$, America).

The concentration of AuNSs in the liposomal solution was determined using inductively coupled plasma spectrometer (ICP, Prodigy ICP Spectrometer). Briefly, $100 \mu \mathrm{L}$ AuNS-liposome complex or Dox-AuNS-liposome complex solutions after purification were digested using $250 \mu \mathrm{L}$ aqua-regia overnight. The digested samples were then diluted to $10 \mathrm{~mL}$ using DI water and the gold content was measured by ICP Spectrometer. Gold standard solution was used to construct the standard curve.

\section{Photoacoustic measurement}

The setup for the photoacoustic system is depicted in Supplementary information (Fig. S1). Pulsed laser diode (QD-Q1910-SA-TEC, Quantel, France) operated at 803 $\mathrm{nm}$ was the light source with a pulse width of $136 \mathrm{~ns}$ and a pulse energy of $1.4 \mathrm{~mJ}$ at $7 \mathrm{kHz}$ repetition rate. A ground glass was used in front of the laser window to make the laser beam more uniform. The sample was placed inside a water bath. The liposome solution and/or tissue sample was irradiated with the homogeneous laser beam and the photoacoustic signals were collected by the ultrasound transducer. A single element ultrasound transducer (V323-SU/2.25 MHz, Olympus NDT) with $2.25 \mathrm{MHz}$ central frequency, $13 \mathrm{~mm}$ active area, and $70 \%$ nominal bandwidth was used for all the photoacoustic experiments. The photoacoustic signals were subsequently amplified, and band pass was filtered by an Amplifier/Filter (A/F) unit (Olympus-NDT, 5072PR). Finally, the output from A/F unit was digitized with a data acquisition card (GaGe, compuscope 4227) and stored in the computer. For photoacoustic spectrum measurement, an optical parametric oscillator (Continuum, Surelite OPO) system pumped by a $532 \mathrm{~nm}$ Nd:YAG laser (Continuum, Surelite Ex) was used. The laser wavelength was varied from 660 to $900 \mathrm{~nm}$ to obtain the photoacoustic spectrum.

\section{Laser triggered Dox release from Dox-AuNS-liposome complex}

$50 \mu \mathrm{L}$ of Dox-AuNS-liposome complex was placed on a microscopic slide, two centimetres away from the laser window. The sample was irradiated with the pulsed laser at $803 \mathrm{~nm}$ for varying time points $(0-10 \mathrm{~s})$ with $5 \mathrm{~mm}$ laser spot and the output energy of $1.4 \mathrm{~mJ} \mathrm{~cm}^{-2}$. The solution could be collected after each cycle and was replaced with fresh aliquots. The collected samples were diluted in DI water and were centrifuged at $10,000 \times g$ to remove the DoxAuNS-liposome complex. The supernatant containing released Dox was quantified by measuring the absorbance of Dox at $480 \mathrm{~nm}$ using a UV-vis spectrophotometer (Spectra$\max )$. The same protocol was carried for the Dox-liposome complex without AuNSs.

For in vitro drug release experiments, B16 F10 melanoma cells (ATCC) were seeded on a 48-well plate overnight before being incubated with Dulbecco's Modified Eagle medium (DMEM) containing 10\% FBS and the liposome samples. Free Dox and AuNSs acted as controls. After 24 $\mathrm{h}$ of labelling, the liposome-containing medium was replaced with fresh one. The cells were immediately treated with $803 \mathrm{~nm}$ pulse laser for $120 \mathrm{~s}$ with the energy of 1.4

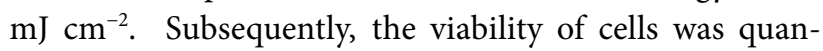
tified using Alamar Blue and the results were expressed as the percentage of viable cells as against the untreated cells. Alongside, live/dead staining was performed using calcein acetomethoxy (CAM) and propidium iodide (PI) for qualitative analysis of the effect of laser treatment. Upon exposure to laser, the cells were labelled with CAM $\left.(2 \mu \mathrm{mol} \mathrm{L})^{-1}\right)$ and PI $\left(4 \mu \mathrm{mol} \mathrm{L}^{-1}\right)$ in DMEM without phenol red solution. Following $30 \mathrm{~min}$ incubation, the cells were washed and rinsed with phosphate-buffered saline before being placed in DMEM without phenol red. The cells were then imaged using LX71 inverted fluorescence microscope (Olympus) equipped with a Retiga-2000R CCD camera. The images were captured at $20 \times$ image magnification with an exposure time of $50 \mathrm{~ms}$ and a gain number of 7 . Finally, the images were processed to normalize the background colour using ImageJ software.

\section{RESULTS}

\section{Synthesis and characterization of AuNS-liposome complex}

$\mathrm{HAuCl}_{4}$ containing liposomes were prepared by hydrating the lipid film with $20 \mathrm{mmol} \mathrm{L}^{-1} \mathrm{HAuCl}_{4}$ aqueous solution (Scheme 1). After removing the free $\mathrm{HAuCl}_{4}$, the liposomes were reconstituted with $1 \mathrm{~mol} \mathrm{~L}^{-1}$ HEPES solution for one hour in the dark. Later, the HEPES buffer was replaced with DI water by using gel filtration chromatography. The as-synthesized liposomes had a zeta potential of $-35.33 \pm 1.53 \mathrm{mV}$. The as-synthesized liposomes had a hydrodynamic diameter of $422.6 \pm 0.9 \mathrm{~nm}$ (Fig. S2a). However, the size increased during the subsequent storage, especially after 14 days (Fig. S2b). Thus all the experiments in this report were done with freshly prepared liposomes 
within 3 days. Their maximum UV-vis absorbance is at 803 $\mathrm{nm}$ (Fig. 1a), which falls within the 'optical window' in biological tissues $[15,16]$. When the liposomes were examined under TEM, AuNSs are seen on the lipid memebrane of liposomes (Fig. 1b). After being extracted from the liposomes and examined under HRTEM, these AuNSs with branches have the similar irregular shape with the diameter of approximately $44 \mathrm{~nm}$ (Fig. 1c).

\section{AuNS-liposome complex as contrast agents for photoacoustic imaging}

The photoacoustic spectrum of AuNS-liposome complex was collected as the laser was continuously tuned from 660 to $900 \mathrm{~nm}$ in a $10 \mathrm{~nm}$ increment. As shown in Fig. 2a, the complex shows the strongest photoacoustic contrast between 750 and $870 \mathrm{~nm}$. It is noted that this laser scanning did not change the absorption of AuNS-liposomes. Taking $803 \mathrm{~nm}$ as the preferred wavelength, we further studied the relation between the liposome concentration and photoacoustic signal. As shown in Fig. 2b, there is a linear relationship between the photoacoustic signal strength and the concentration of liposomes.

The photoacoustic signals were further recorded from both liposome samples and fresh blood (from mice). Blood being an intrinsic absorber of light produces photoacoustic signals and therefore, any potential contrast agent is usually compared against the blood for their potency [17-19]. Since the PA signal of AuNS-liposomes was related with their concentration, a series of PA signal was collected (Table $\mathrm{S} 1$ and Fig. 2b). Clearly, $2 \mathrm{mg} \mathrm{mL}^{-1}$ was the minimal concentration for the identification of AuNS-liposomes from the blood. Fig. 2c shows the direct photoacoustic signal comparison between the liposomes $\left(2 \mathrm{mg} \mathrm{mL}^{-1}\right)$ and blood. The peak-to-peak photoacoustic signal from AuNS-liposome complex was $675.9 \mathrm{mV}$ while that of blood was $456.8 \mathrm{mV}$. AuNS-liposome complex showed almost 1.5 fold increase in the photoacoustic signals compared to that of blood. Excitingly, this signal difference was not significantly influenced when they were placed within $1-\mathrm{cm}$ deep inside chicken breast tissue. As shown in Fig. 2d, AuNS-liposome complex shows a peak-to-peak photoacoustic signal of $108.6 \mathrm{mV}$ when being placed under $1-\mathrm{cm}$ fresh chicken breast tissue while blood produces $66 \mathrm{mV}$. In this case, peak-to-peak photoacoustic signal intensity of AuNS-liposome complex is still $\sim 1.5$ of that of blood.

Thus the photoacoustic contrast brought by AuNS-liposome complex could be maintained after the filtration of tissue, which suggests that it can be used as a contrast agent for the photoacoustic imaging applications.

\section{Controlled drug release from AuNS-liposome complex}

To explore the utilization of AuNS-liposome complex for drug delivery, Dox was encapsulated within the lipid bilayer of the AuNS-liposome complex to form Dox-AuNS-liposome complex. In order to facilitate the encapsulation of Dox in lipid bilayer, TEA was used to deprotonate Dox- $\mathrm{HCl}$ to the hydrophobic Dox $[13,20]$. For Dox-liposome complex, DI water instead of $\mathrm{HAuCl}_{4}$ was used during the synthesis.

The concentration of Dox within the liposomes was
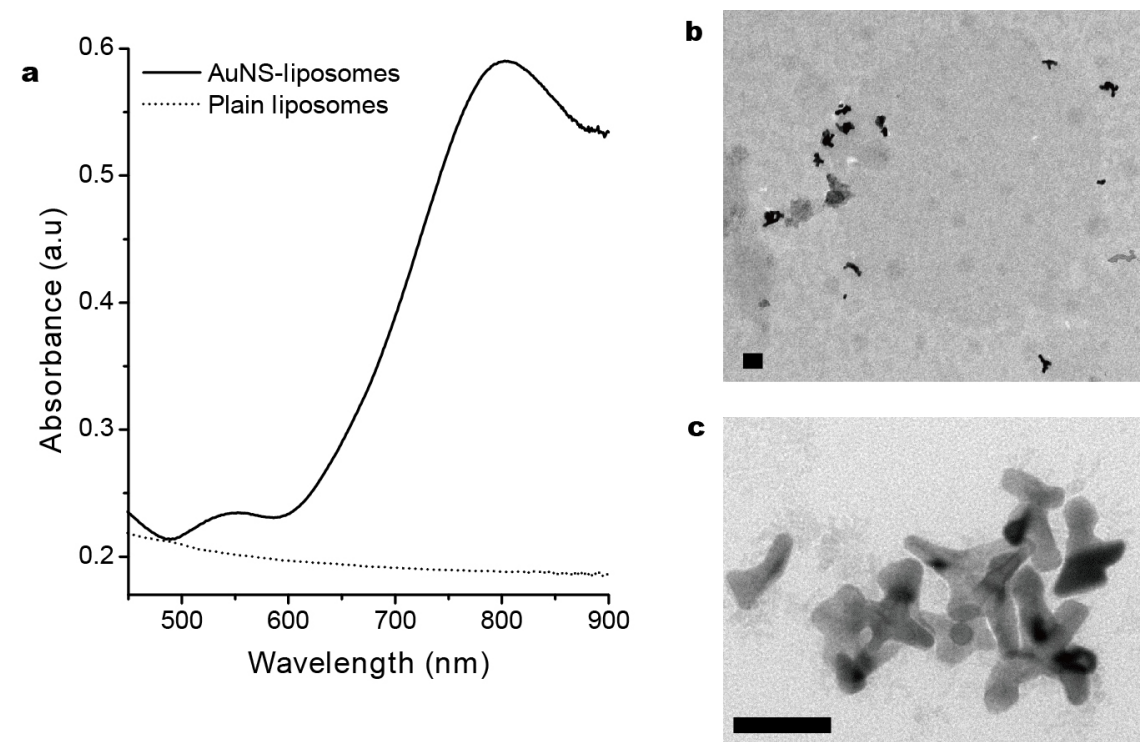

Figure 1 Characterization of AuNS-liposomes: (a) UV spectrum of plain liposomes (dotted line) and AuNS-liposome complex (solid line). (b) TEM image of AuNS-liposomes. Scale bar: $50 \mathrm{~nm}$; (c) HRTEM image of AuNSs collected from AuNS-liposomes. Scale bar: $50 \mathrm{~nm}$. 

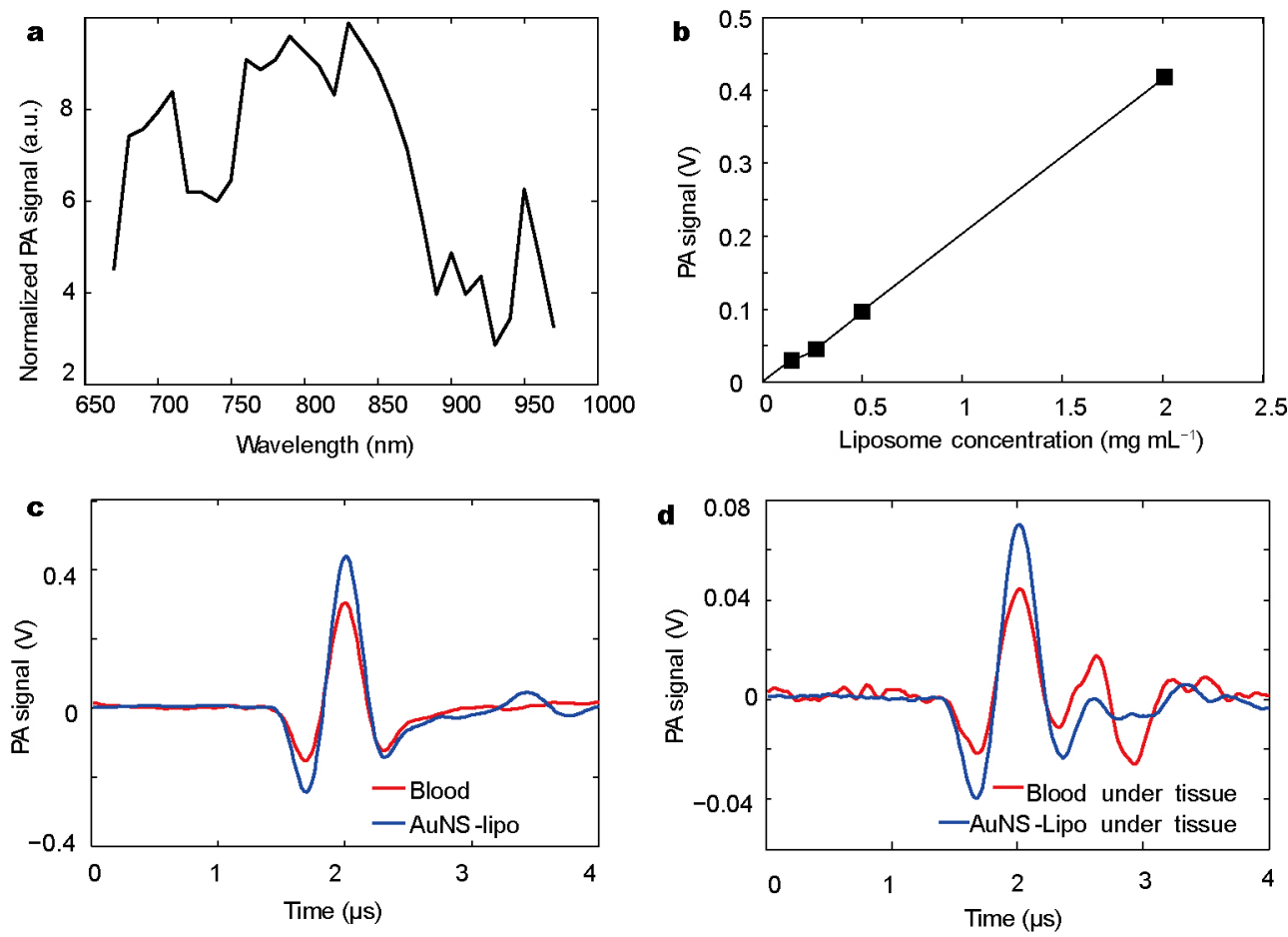

Figure 2 AuNS-liposome complex as contrast agents for photoacoustic imaging: (a) photoacoustic spectra of AuNS-liposome complex; (b) photoacoustic signal intensity $v s$. concentration of AuNS-liposome complex; (c) comparison of photoacoustic signals of $2 \mathrm{mg} \mathrm{mL} \mathrm{m}^{-1}$ AuNS-liposome complex and fresh mouse blood; (d) comparison of photoacoustic signals of $2 \mathrm{mg} \mathrm{mL}^{-1}$ AuNS-liposome complex and fresh mouse blood under 1-cm deep fresh chicken breast tissue. The excitation laser pulse was at $803 \mathrm{~nm}$ in (b-d).

$\sim 10 \%$ by weight, which was calculated by digesting the Dox-loaded liposomes using Triton X-100 and measuring its absorbance at $480 \mathrm{~nm}$. Formation of AuNSs in the Dox-AuNS-liposome was firstly confirmed through the change in the maximal peak absorbance in the UV-vis spectrum (Fig. 3a). Different from Dox-liposomes that show the highest absorbance at $480 \mathrm{~nm}$, the maximal absorbance of Dox-AuNS-liposomes shows up at $650 \mathrm{~nm}$. Clearly this NIR absorption was due to the appearance of AuNSs. The formation of AuNSs was further confirmed by lysing the liposomes and examining the extracted AuNSs through TEM (Fig. S3a). DLS study also revealed that the presence of AuNSs did not significantly change the size of the liposomes $(129.3 \pm 1.6 \mathrm{~nm}$ vs. $115.9 \pm 2.5 \mathrm{~nm}$, Fig. S3b). We further examined the photoacoustic spectra of Dox-AuNS-liposomes that presented their strongest signal between $660 \mathrm{~nm}$ and $750 \mathrm{~nm}$ (Fig. S3c).

Later, Dox-AuNS-liposome solution was exposed to the NIR pulsed laser for varying time points ( $0-10$ seconds) with an energy density of $1.4 \mathrm{~mJ} \mathrm{~cm}^{-2}$. As shown in Fig. 3b, the presence of AuNS enables 100\% release of encapsulated Dox from the liposomes within only 10 seconds under the laser exposure while the Dox-liposomes have only $~ 10 \%$ release under the same experimental condition.

\section{Cell cytotoxicity assay with Dox-AuNS-liposomes}

To examine the potential therapeutic effectiveness of Dox-AuNS-liposome complex, the melanoma cells were incubated with AuNS-liposomes, Dox-liposomes, or Dox-AuNS-liposomes with or without the treatment of pulsed laser. Later the cell viability was quantified using Alamar Blue assay and was normalized against the viability of the untreated cells. Dox was maintained at $\sim 30 \mu \mathrm{g}$ $\mathrm{mL}^{-1}$ for the Dox-containing groups and Au concentration was kept at $70 \mu \mathrm{mol} \mathrm{L}^{-1}$ for AuNSs containing samples. As shown in Fig. 4a, there is negligible cell death for all samples without the treatment of pulsed laser, except the Dox treated groups. However, if the pulsed laser was applied, AuNS-liposome complex and Dox-AuNS-liposome complex resulted in approximately $82.42 \pm 5.23 \%$ and $93.75 \pm 5.37 \%$ of cell death. Besides Alamar blue assay, live/dead assay was also performed with CAM and PI (Fig. $4 \mathrm{~b})$, in which the results echoed the Alamar Blue viability assay.

\section{DISCUSSION}

HEPES is a moderate reducing agent for the synthesis of gold nanoparticles with different morphologies $[9,10]$. The piperazine ring in the HEPES is responsible for the reduc- 

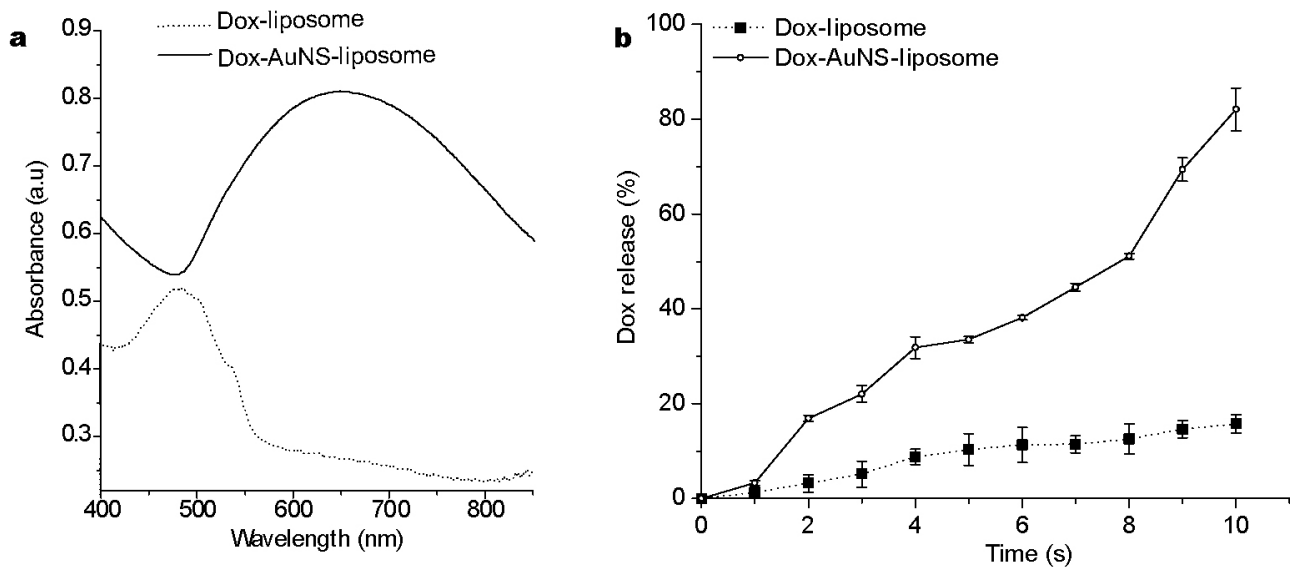

Figure 3 AuNS-liposome complex as the drug carrier for controlled release: (a) UV-vis spectra of Dox-liposomes and Dox-AuNS-liposome complex; (b) release of Dox from the Dox-liposomes and Dox-AuNS-liposome complex under the irradiation of NIR pulse laser. Error bars indicate the standard deviation (SD, $N \geq 3$ ).

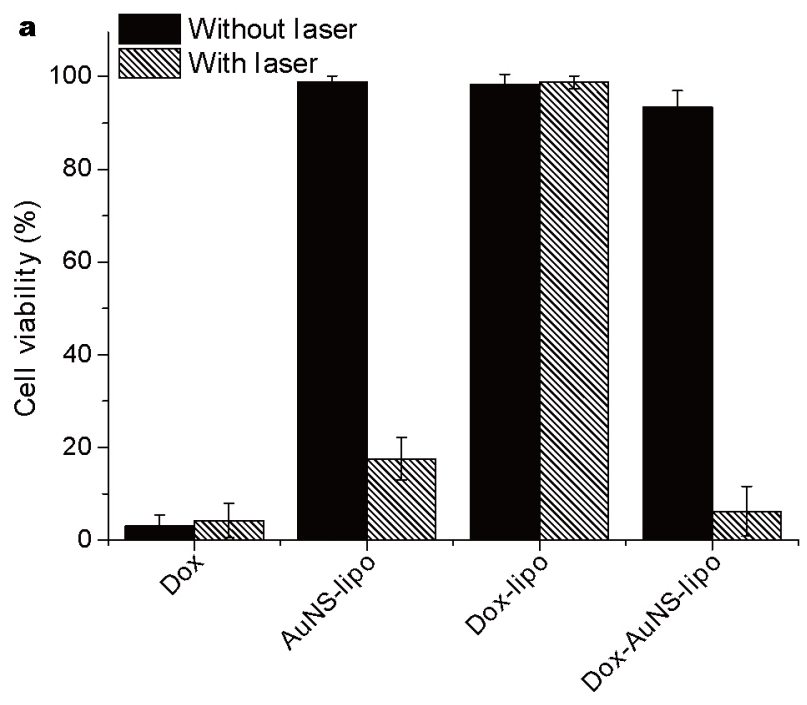

b
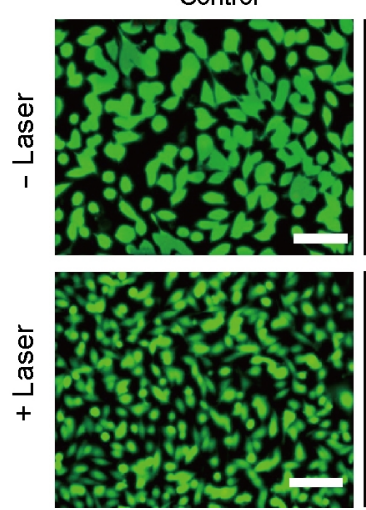

Dox
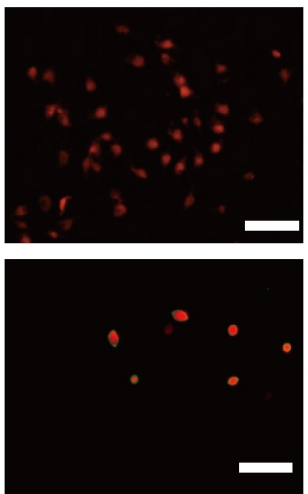

AuNS-liposome
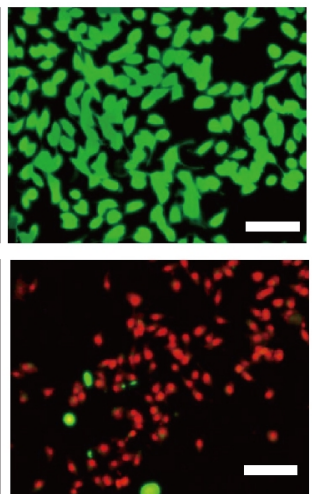

Dox-liposome

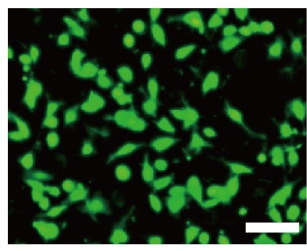

Dox-AuNS-liposome
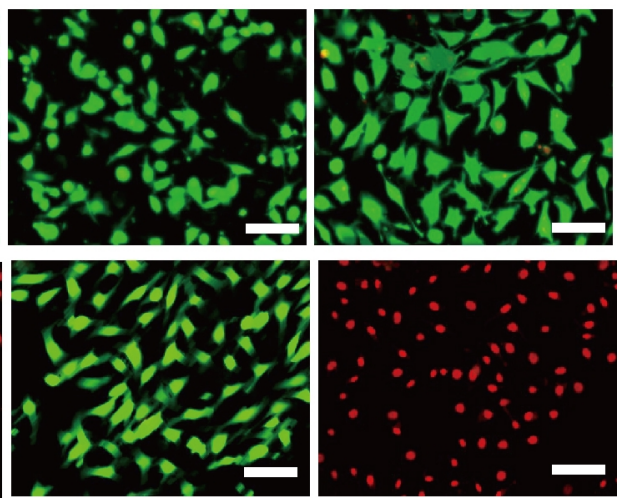

Figure 4 Viability of B16-F10 melanoma cells after the treatment with Dox, AuNS-liposomes, Dox-liposomes, and Dox-AuNS-liposomes with or without pulsed laser: (a) Alamar Blue cell viability assay. Error bars indicate the SD $(N \geq 3)$; (b) live/dead staining assay with calcein acetomethoxy and propidium iodide. Scale bar: $100 \mu \mathrm{m}$. 
tion of $\mathrm{HAuCl}_{4}$ from $\mathrm{Au}^{3+}$ to $\mathrm{Au}^{0}$. In this study, we explored the use of HEPES to reduce $\mathrm{HAuCl}_{4}$ that was pre-encapsulated within liposomes. The key is whether HEPES could diffuse through the lipid bilayer of liposomes and whether the $\mathrm{HAuCl}_{4}$ concentration encapsulated in liposomes was sufficient for the formation of AuNSs. The concentrations of $\mathrm{HAuCl}_{4}\left(20 \mathrm{mmol} \mathrm{L}^{-1}\right)$ and HEPES $\left(1 \mathrm{~mol} \mathrm{~L}^{-1}\right)$ during the synthesis were selected based on the early work by Xie and Maiorano et al. $[9,21]$. Although the diffusion process could not be directly observed, the successful formation of AuNSs on the lipid membrane of liposomes suggested both conditions were met (Figs $1 b$ and c). The shape of gold nanoparticles could be controlled through tuning the ratio between $\mathrm{HAuCl}_{4}$ and HEPES. However, considering their maximal absorption in the NIR range, AuNSs were made.

Surface-plasmon resonance active nanoparticles such as Au nanoparticles are able to generate photoacoustic signals when being irradiated with a pulsed laser and thus can provide contrast in photoacoustic imaging [22-25]. The incident pulsed laser is absorbed by Au nanoparticles and converted to heat. Then the thermal energy causes a thermoelastic expansion resulting in pressure wave. The pressure wave is recorded as photoacoustic signal by an ultrasound transducer. We first examined the photoacoustic spectrum of AuNS-liposomes (Fig. 2a). From 660 to $900 \mathrm{~nm}$, the complex shows the strongest photoacoustic contrast between 750 and $870 \mathrm{~nm}$. Fig. 1a shows that AuNS-liposomes have a maximal absorbance at $803 \mathrm{~nm}$ in UV-vis spectrum. Therefore, pulsed diode laser at $803 \mathrm{~nm}$ was chosen as the laser source for further experiments.

As expected, the photoacoustic signal intensity has a linear relation with the Au concentration (Fig. 2b). Moreover, the peak-to-peak photoacoustic signal amplitude was higher than that of blood even under $1-\mathrm{cm}$ deep chicken breast tissue. This confirms that for in vivo photoacoustic imaging applications AuNS-liposome complex can be used as a potential photoacoustic contrast agent.

Recently, we demonstrated that pulsed laser could disrupt liposomes containing Au nanoparticles through microbubble cavitation, which resulted in the release of encapsulated drugs [7]. We believe that a similar phenomenon would be observed with the AuNS-liposome complex. As a proof-of-concept, Dox was incorporated within the lipid bilayer of the liposomes. Although the formation of AuNSs in the Dox-AuNS-liposome complex is evident from the UV-vis absorption spectra (Fig. 3a) and the TEM examination (Fig. S3a), there is a blue-shift of the absorption spectrum of the Dox-AuNS-liposomes (Fig. S3d), when compared to that of AuNS-liposomes. This could be attributed to the morphological change of the AuNSs and further studies are required to understand this phenomenon with more details. In addition, the characteristic peak of Dox at $480 \mathrm{~nm}$ is not observed in the Dox-AuNS-liposomes (Fig. 3a). This effect may be due to the negligible or very weak absorption of the Dox or dominant effect of the peak of the AuNSs present in the complex [26].

The synthesized Dox-AuNS-liposomes were then irradiated with the pulsed laser at $803 \mathrm{~nm}$ where Dox-AuNS-liposomes show good absorption (Fig. 3b). Complete release of Dox from Dox-AuNS-liposome complex was achieved within $10 \mathrm{~s}$. However, there were only $\sim 10 \%$ Dox released from the Dox-liposomes under the same condition. This clearly indicates that the Dox release from Dox-AuNS-liposome complex was due to the laser stimulation that might execute through heat or microbubble formation.

Finally, the therapeutic effect of Dox-AuNS-liposome complex was explored with melanoma cells (Fig. 4). Compared with AuNS-liposomes, Dox-liposomes, and Dox-AuNS-liposomes, Dox shows consistent cytotoxicity under both conditions. In contrast, Dox-liposomes did not have much effect on the cell viability. This is because the liposomes have a phase transition temperature at $40.8^{\circ} \mathrm{C}$ [7] and it has been reported that the cytotoxicity of the drug encapsulated in temperature-sensitive liposomes is highly reduced when maintained at $37^{\circ} \mathrm{C}[27,28]$. AuNS-liposomes are non-toxic without laser treatment. However, the exposure to NIR laser dramatically changes their cytotoxicity, which should be due to the cavitation induced by the microbubble formation due to the transient heating of AuNS-liposomes [29-31]. As a combination of Dox-liposomes and AuNS-liposomes, Dox-AuNS-liposome complex shows similar non-toxic effects without laser treatment. However, with the treatment of laser, it also shows the highest cytotoxicity due to the synergetic effect of local heating, microbubble cavitation and Dox.

\section{CONCLUSION}

In summary, this report introduces the synthesis of AuNSs containing liposomes by the in situ reduction of the gold precursor $\mathrm{HAuCl}_{4}$ through HEPES diffusion and reduction. It allows the formation and simultaneous encapsulation of AuNSs within liposomes. Due to the presence of AuNSs, these liposomes can produce strong photoacoustic signals in the NIR region even at $1 \mathrm{~cm}$ deep inside biological tissue. Furthermore, when there are drugs (i.e., Dox) within these liposomes, the irradiation with the NIR pulse laser can disrupt the liposomes and trigger the release of these pre-encapsulated drugs to perform the local 
treatment. We believe this platform has the potential as a dual-modal theranostic agent since it can be used for both imaging as well as therapy with controlled drug delivery and targeting. In the future studies, we will test the capability further with an in vivo small animal model.

Received 18 July 2016; accepted 29 August 2016; published online 8 October 2016

1 Alvarez-Lorenzo C, Bromberg L, Concheiro A. Light-sensitive intelligent drug delivery systems. Photochem Photobiol, 2009, 85: 848-860

2 Movahedi F, Hu RG, Becker DL, et al. Stimuli-responsive liposomes for the delivery of nucleic acid therapeutics. Nanomed Nanotechnol Biol Med, 2015, 11: 1575-1584

3 Leung SJ, Romanowski M. Light-activated content release from liposomes. Theranostics, 2012, 2: 1020-1036

4 Pashkovskaya A, Kotova E, Zorlu Y, et al. Light-triggered liposomal release: membrane permeabilization by photodynamic action. Langmuir, 2010, 26: 5726-5733

5 Lozano N, Al-Jamal WT, Taruttis A, et al. Liposome-gold nanorod hybrids for high-resolution visualization deep in tissues. J Am Chem Soc, 2012, 134: 13256-13258

6 Taruttis A, Lozano N, Nunes A, et al. siRNA liposome-gold nanorod vectors for multispectral optoacoustic tomography theranostics. Nanoscale, 2014, 6: 13451-13456

7 Mathiyazhakan M, Yang Y, Liu Y, et al. Non-invasive controlled release from gold nanoparticle integrated photo-responsive liposomes through pulse laser induced microbubble cavitation. Colloid Surf B-Biointerfaces, 2015, 126: 569-574

8 Akbarzadeh A, Rezaei-Sadabady R, Davaran S, et al. Liposome: classification, preparation, and applications. Nanoscale Res Lett, 2013, 8: 102

9 Xie J, Lee JY, Wang DIC. Seedless, surfactantless, high-yield synthesis of branched gold nanocrystals in HEPES buffer solution. Chem Mater, 2007, 19: 2823-2830

10 Chen R, Wu J, Li H, et al. Fabrication of gold nanoparticles with different morphologies in HEPES buffer. Rare Met, 2010, 29: 180-186

11 Dua JS, Rana AC, Bhandari AK. Liposome: methods of preparation and applications. Int J Pharm Sci Res, 2012, 3: 14-20

12 Szoka F, Papahadjopoulos D. Comparative properties and methods of preparation of lipid vesicles (liposomes). Annu Rev Biophys Bioeng, 1980, 9: 467-508

13 Missirlis D, Kawamura R, Tirelli N, et al. Doxorubicin encapsulation and diffusional release from stable, polymeric, hydrogel nanoparticles. Eur J Pharm Sci, 2006, 29: 120-129

14 Genç R, Ortiz M, O’Sullivan CK. Diffusion-controlled synthesis of gold nanoparticles: nano-liposomes as mass transfer barrier. J Nanopart Res, 2014, 16: 2329

15 Anderson RR, Parrish JA. The optics of human skin. J Investig Dermatol, 1981, 77: 13-19

16 Tsai CL, Chen JC, Wang WJ. Near-infrared absorption property of biological soft tissue constituents. J Med Biol Eng, 2001, 21: 7-14

17 Upputuri PK, Pramanik M. Pulsed laser diode based optoacoustic imaging of biological tissues. Biomed Phys Eng Express, 2015, 1: 045010

18 Huang S, Upputuri PK, Liu H, et al. A dual-functional benzobisthiadiazole derivative as an effective theranostic agent for nearinfrared photoacoustic imaging and photothermal therapy. J Mater
Chem B, 2016, 4: 1696-1703

19 Lu W, Huang Q, Ku G, et al. Photoacoustic imaging of living mouse brain vasculature using hollow gold nanospheres. Biomaterials, 2010, 31: 2617-2626

$20 \mathrm{Xu}$ J, Zhao Q, Jin Y, et al. High loading of hydrophilic/hydrophobic doxorubicin into polyphosphazene polymersome for breast cancer therapy. Nanomed Nanotechnol Biol Med, 2014, 10: 349-358

21 Maiorano G, Rizzello L, Malvindi MA, et al. Monodispersed and size-controlled multibranched gold nanoparticles with nanoscale tuning of surface morphology. Nanoscale, 2011, 3: 2227-2232

22 Liu Y, Yuan H, Fales A, et al. Multifunctional gold nanostars for molecular imaging and cancer therapy. Front Chem, 2015, 3: 51

23 Bao C, Conde J, Pan F, et al. Gold nanoprisms as a hybrid in vivo cancer theranostic platform for in situ photoacoustic imaging, angiography, and localized hyperthermia. Nano Res, 2016, 9: 1043-1056

24 Jiang Y, Deng Z, Yang D, et al. Gold nanoflowers for 3D volumetric molecular imaging of tumors by photoacoustic tomography. Nano Res, 2015, 8: 2152-2161

25 Mou J, Chen Y, Ma M, et al. Facile synthesis of liposome/ $\mathrm{Cu}_{2-x} \mathrm{~S}$ based nanocomposite for multimodal imaging and photothermal therapy. Sci China Mater, 2015, 58: 294-301

26 Rai VN, Srivastava AK, Mukherjee C, et al. Surface enhanced absorption and transmission from dye coated gold nanoparticles in thin films. Appl Opt, 2012, 51: 2606-2615

27 Ponce AM, Viglianti BL, Yu D, et al. Magnetic resonance imaging of temperature-sensitive liposome release: drug dose painting and antitumor effects. J Natl Cancer Institute, 2007, 99: 53-63

28 Kim MS, Lee DW, Park K, et al. Temperature-triggered tumor-specific delivery of anticancer agents by cRGD-conjugated thermosensitive liposomes. Colloid Surf B Biointerfaces, 2014, 116: 17-25

29 Lapotko DO, Lukianova E, Oraevsky AA. Selective laser nano-thermolysis of human leukemia cells with microbubbles generated around clusters of gold nanoparticles. Lasers Surg Med, 2006, 38: 631-642

30 Lukianova-Hleb EY, Belyanin A, Kashinath S, et al. Plasmonic nanobubble-enhanced endosomal escape processes for selective and guided intracellular delivery of chemotherapy to drug-resistant cancer cells. Biomaterials, 2012, 33: 1821-1826

31 Hleb EY, Hafner JH, Myers JN, et al. LANTCET: elimination of solid tumor cells with photothermal bubbles generated around clusters of gold nanoparticles. Nanomedicine, 2008, 3: 647-667

Acknowledgments The work was partially supported by NTU-Northwestern Institute for Nanomedicine (To Xu CJ), the Tier-2 Grant funded by the Ministry of Education in Singapore (ARC2/15: M4020238 to M.P.). Dhayani A thanks UGC for junior research fellowship. Vemula PK thanks DBT for Ramalingaswami ReEntry fellowship. We appreciate Mr. Stanley Sim Siong Wei to help us draw the illustration.

Author contributions Mathiyazhakan $\mathrm{M}$ and $\mathrm{Xu} \mathrm{C}$ conceived the project and wrote the manuscript. Mathiyazhakan $\mathrm{M}$ and Upputuri PK performed the cell culture, liposome synthesis and experiments. Upputuri PK, Sivasubramanian K, Pu K and Pramanik M assisted and provided constructive input for NIR laser stimulation experiments. Dhayani A, Vemula PK, Zou P and Yang C performed and provided inputs on TEM analysis. The manuscript was written through contributions of all authors. All authors have given approval to the final version of the manuscript.

Conflict of interest The authors declare that they have no conflict of interest. 
Supplementary information Supplementary data are available in the online version of the paper.
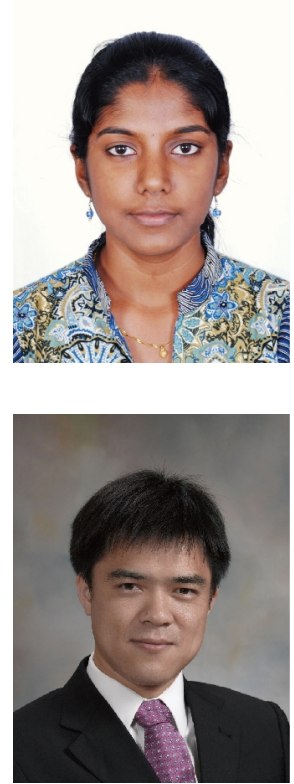

Malathi Mathiyazhakan studied industrial biotechnology at SASTRA University, India. As an undergraduate, she conducted research on host:virus interactions with Chikungunya virus. After receiving her B.Tech. in Industrial Biotechnology in 2011, she joined Nanyang Technological University (Singapore) as a PhD student under the supervision of Prof. Chenjie $\mathrm{Xu}$. Her research interest lies in the area of smart nanomaterials for drug delivery and imaging. Current work focuses on the facile synthesis of gold nanoparticles containing liposomes for drug delivery and photoacoustic imaging.

Chenjie Xu is an assistant professor at Nanyang Technological University. He is interested in the development of patientfriendly and efficacious nanotechnologies for drug delivery. The key in this process is to address the unmet need in the drug administration with innovative strategies that can be quickly translated from bench to bedside. Currently, his laboratory is interested in developing nanotechnologies for the targeted drug delivery in the most common diseases like cancer and scar formation.

\section{脂质体内金纳米星的原位合成以及在药物释放和光声造影上的应用}

Malathi Mathiyazhakan ${ }^{1}$, Paul Kumar Upputuri ${ }^{1}$, Kathyayini Sivasubramanian ${ }^{1}$, Ashish Dhayani ${ }^{2}$, Praveen Kumar Vemula ${ }^{2,3}$, 邹培超 ${ }^{4}$, 浦㑆裔 ${ }^{1}$ 杨诚 ${ }^{4}$, Manojit Pramanik ${ }^{1}$, 徐臣杰 ${ }^{1,5^{*}}$

摘要 本文通过在脂质体内还原金的前体 $\mathrm{HAuCl}_{4}$ 原位合成了金纳米星. 这种设计跟常用的在脂质体内装载金纳米球的方法相比, 优点是方 便快捷的同时实现了金纳米材料的形成和装载. 通过改变实验条件, 合成的金纳米星具有可控的尺寸, 以及在可见区到近红外区之间的可 控吸收光谱. 作为一个例子, 我们在脂质体内合成了最大吸收在 $803 \mathrm{~nm}$ 的金纳米星. 这种材料具有温度敏感性, 在近红外区可以产生比血液 好1.5倍的光声造影信号. 当我们把抗癌药物阿霉素装载到这种脂质体内时, 近红外区的激光照射可以在 10 秒内触发药物 $100 \%$ 的释放. 相 对应的, 不含纳米星的脂质体在同等条件下只能释放 $10 \%$ 的药物, 也不具备光声造影的信号增强. 最后, 我们在癌细胞内测试了该脂质体 的疗效, 初步验证了该体系的应用前景. 